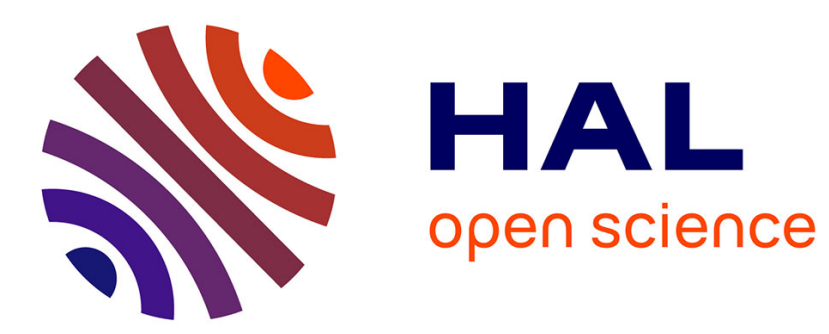

\title{
Role of convective acceleration in the interfacial instability of liquid-gas coaxial jets
}

Guillaume Ricard, Nathanaël Machicoane, Rodrigo Osuna-Orozco, Peter D

Huck, Alberto Aliseda

\section{- To cite this version:}

Guillaume Ricard, Nathanaël Machicoane, Rodrigo Osuna-Orozco, Peter D Huck, Alberto Aliseda. Role of convective acceleration in the interfacial instability of liquid-gas coaxial jets. Physical Review Fluids, 2021, 6, 10.1103/physrevfluids.6.084302 . hal-03318040

\section{HAL Id: hal-03318040 \\ https://hal.science/hal-03318040}

Submitted on 9 Aug 2021

HAL is a multi-disciplinary open access archive for the deposit and dissemination of scientific research documents, whether they are published or not. The documents may come from teaching and research institutions in France or abroad, or from public or private research centers.
L'archive ouverte pluridisciplinaire HAL, est destinée au dépôt et à la diffusion de documents scientifiques de niveau recherche, publiés ou non, émanant des établissements d'enseignement et de recherche français ou étrangers, des laboratoires publics ou privés. 


\title{
Role of convective acceleration in the interfacial instability of liquid-gas coaxial jets
}

\author{
Guillaume Ricard, ${ }^{1}$ Nathanaël Machicoane $\odot{ }^{2,},{ }^{*}$ Rodrigo Osuna-Orozco $\bullet,{ }^{1}$ \\ Peter D. Huck, ${ }^{1}$ and Alberto Aliseda ${ }^{1}$ \\ ${ }^{1}$ University of Washington, Department of Mechanical Engineering, Seattle, Washington 98105, USA \\ ${ }^{2}$ Univ. Grenoble Alpes, CNRS, Grenoble INP, LEGI, 38000 Grenoble, France
}

(Received 29 January 2021; accepted 23 July 2021; published 6 August 2021)

\begin{abstract}
Interfacial instabilities play a major role in breakup events in turbulent multiphase flow. Their role has been clearly identified for two-fluid atomization, and is of paramount importance in spray formation. In planar geometries, Kelvin-Helmholtz instabilities are the main mechanism of creation of a two-phase mixing layer, and information such as wavelengths and frequencies is available in the literature. In cylindrical geometries, the instabilities quickly become three-dimensional and thorough characterization is lacking, despite a wide range of applications using coaxial atomization. We conduct an experimental study of how the interfacial instabilities of a liquid jet surrounded by a turbulent gas co-flow accelerate and develop, before break-up and spray formation. We use high-speed shadowgraphy over a wide range of gas Reynolds numbers to compute the velocity of interfacial perturbations, using Lagrangian tracking, followed by a Eulerian conditioning to obtain local statistics. We identified two regimes of the gradient of the longitudinal mean velocity as a function of the gas Reynolds number: a quadratic scaling at low gas Reynolds numbers and a linear scaling at higher gas Reynolds number. In contrast, the transverse velocity gradients show a linear scaling with gas Reynolds number throughout the studied range.
\end{abstract}

DOI: 10.1103/PhysRevFluids.6.084302

\section{INTRODUCTION}

Interfacial instabilities are of paramount importance in multiphase flows and specifically in the formation of sprays [1]. As in other widespread applications, interfacial instability initiation and growth rate are the leading factors in this physical phenomenon. Generally speaking, in fluid mechanics, an interface can separate two streams of the same fluid that flow at different velocities, two miscible fluids with different densities or viscosities, or two immiscible fluids. Atomization phenomena occur in many situations in nature (exchange between ocean and atmosphere [1]) and are widely used in industry (propulsion and combustion [2,3], spray drying, food processing). It can also be applied in the understanding of fundamental physical mechanisms at play in other fields, such as health care or meteorology [1].

Instabilities can appear and evolve along such interfaces [4,5]. For example, the KelvinHelmholtz instability develops along a sheared interface, and grows at a distinct wavelength that has been predicted theoretically [6,7], and confirmed experimentally [8]. Kelvin-Helmholtz instabilities can appear for example on a surface of water below a flow of air [5,9].

This instability, developing along the intact part of the jet, leads to the primary breakup. Interfacial instabilities in atomization appear because the two different fluids (gas and liquid) move

\footnotetext{
*nathanael.machicoane@univ-grenoble-alpes.fr
} 
at different velocities. Shear-driven (Kelvin-Helmholtz) and acceleration-driven (Rayleigh-Taylor) instabilities have been reported in this context [10-12]. These three-dimensional (3D) two-phase instabilities grow along the jet and become ligaments which can break into droplets and their characteristics, such as wavelength, frequency, or velocity, have been shown to be of paramount importance for atomization $[10,11]$.

In the formation of sprays, the Kelvin-Helmholtz instability grows, nonlinearities appear, and the liquid accelerates significantly, leading to a Raleigh-Taylor-type breakup, controlled by the balance between surface tension, which tries to minimize surface area (energy) and acceleration, which acts to disrupt the liquid surface into small objects with a high surface-to-volume ratio. This balance between inertia and surface tension defines the Weber number We [13]. These instabilities have been studied extensively in planar gas-liquid interfaces [5,14], and their moving speed can be approximated by the Dimotakis convective velocity $U_{c}=\frac{\sqrt{\rho_{l}} U_{l}+\sqrt{\rho_{g}} U_{g}}{\sqrt{\rho_{l}}+\sqrt{\rho_{g}}}$, where $\rho_{i}$ and $U_{i}$ are the density and the velocity of the liquid $(i=l)$ or gas $(i=g)$ [14]. This study focuses on the growth of those instabilities in the case of coaxial liquid (inner core) and gas (outer annulus) jets, a configuration frequently applied for coaxial atomization $[13,15,16]$. Under the right conditions, the instabilities lead to interfacial perturbations that grow along the liquid surface until it breaks into the fine cloud of droplets in a spray $[10,17]$. As the flow develops, two distinct regions can be defined. The intact core and the region where primary breakup is taking place define the spray near-field [18-20]. The region where drops detach from the liquid jet and can break further downstream due to turbulent fluctuations and gas stresses defines the transition from the near to the midfield. Note that the location and extent of this region depend on atomization parameters [21].

The presence of the annular high speed gas jet tends to excite either axisymmetric (quasi-2D) instabilities, referred to as varicose, or localized 3D instabilities [22]. The former are present all around the liquid jet, while the latter are located at one specific azimuthal coordinate, and may be associated with a large-scale sinusoidal instability referred to as flapping of the liquid jet [23]. Note that the latter could also be a helical instability due to a spiraling liquid jet. This whole set of instabilities contributes to the liquid jet destabilization and breakup. A link between the wavelength and frequency of the instabilities and the drops created has been established in the past $[10,24,25]$. However, many questions remain unanswered, especially on the propagation of the interfacial perturbations caused by the instabilities. To the best of our knowledge no studies systematically investigate the displacement of the surface perturbations over a wide range of Reynolds numbers, and one can wonder when they should be treated as waves versus Lagrangian objects. In this paper, we experimentally study the statistics of the velocity and velocity gradient of the interfacial perturbations present along the core of a liquid jet atomized by a gas co-flow. We explore a large range of gas-to-liquid momentum ratios to account for the wide spectrum of different physical mechanisms and regimes of coaxial atomization. From this, we are able to observe that the transverse and longitudinal velocities of instabilities increase linearly with the distance from the nozzle $x$. We are also able to determine the existence of two distinct regimes for the gradient of the longitudinal velocity of the interface perturbations. In the first one, which involves big scales, the gradient evolves quadratically with the gas velocity, while the second one, involving small scales of the turbulence, produces a linear growth of this gradient. The transition between these regimes is related to the scale of the drops resulting from the atomization process.

The outline of the paper is as follows: In Sec. II, we present the experimental setup using shadowgraphy at different levels of magnification to observe the interface at different scales; Sec. III describes the interface instability tracking and the Eulerian conditioning of the instability velocity statistics; in Sec. IV, the different results obtained for the spatial gradient of the velocity are presented; and finally Secs. V and VI present a discussion of the findings, followed by conclusions obtained from this study. 


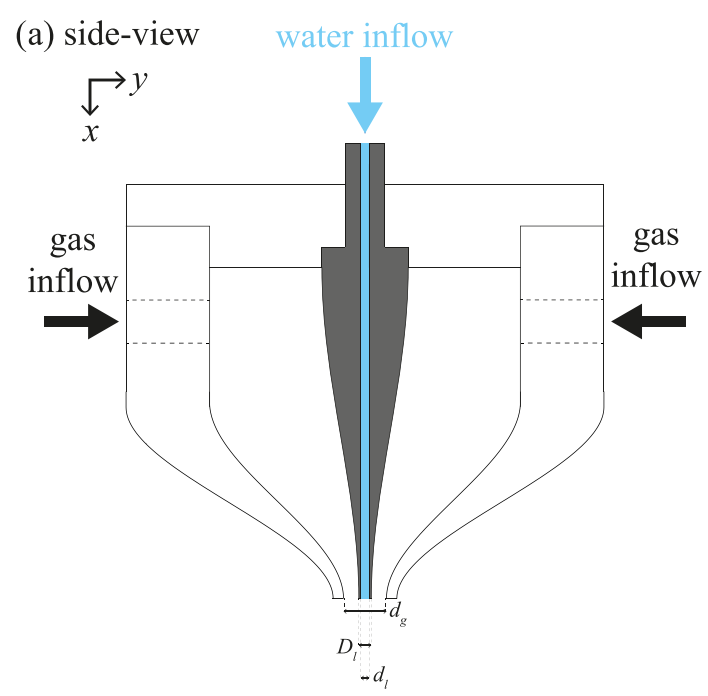

(b) top-view

high-speed
camera

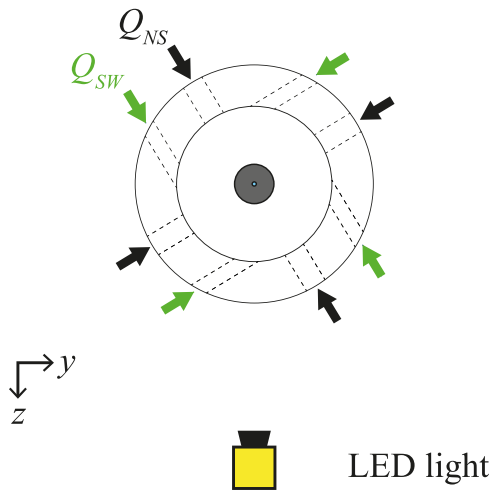

FIG. 1. (a) Axial cut of the coaxial two-fluid atomizer. The liquid jet is surrounded by an air jet which breaks the liquid into droplets. (b) Transverse cut, with black and green arrow, respectively for no-swirl and swirl injection, referring to presence of angular momentum in the gas co-flow.

\section{EXPERIMENTAL SETUP}

\section{A. Canonical atomizer}

The experimental setup consists of a canonical two-fluid coaxial atomizer (see [21,26] for more details). Water flows through a nozzle with an inner diameter $d_{l}=2 \mathrm{~mm}$ and an outer diameter $D_{l}=3 \mathrm{~mm}$. Air flows through a coaxial nozzle with an inner diameter of $d_{g}=10 \mathrm{~mm}$ (Fig. 1), and a gas cross section of $A_{g}=\frac{\pi}{4} d_{\text {eff }}^{2}$, where $d_{\text {eff }}=\sqrt{d_{g}^{2}-D_{l}^{2}} \approx 9.8 \mathrm{~mm}$ (Fig. 1). The flow rates of the two fluids $Q_{l}$ and $Q_{g}$ are controlled and maintained steady thanks to proportional valves and flow meters. The liquid and gas Reynolds numbers $\operatorname{Re}_{l}$ and $\mathrm{Re}_{g}$, defined below, are determined by these controlled flow rates, maintaining the flow regimes in the laminar for the liquid jet and turbulent above the critical mixing transition for the gas jet,

$$
\operatorname{Re}_{l}=\frac{4 Q_{l}}{\pi d_{l} \nu_{l}}, \quad \operatorname{Re}_{g}=\frac{4 Q_{g}}{\sqrt{4 \pi A_{g}} \nu_{g}},
$$

where $v_{l}$ and $v_{g}$ are the kinematic viscosity of the liquid and of the gas, respectively. For this study, the liquid injection is kept at laminar conditions with $\mathrm{Re}_{l}=1172$, while the gas flow rate varied such that $\mathrm{Re}_{g}$ is between 1.41 and $8.36 \times 10^{4}$. Note that changes in the gas Reynolds number are solely achieved here by changes in the gas flow rate.

To quantify the development of the instability between the two fluids, the momentum ratio $M$ or gas-to-liquid dynamic pressure ratio, is used. It is defined as

$$
M=\frac{\rho_{g} U_{g}^{2}}{\rho_{l} U_{l}^{2}},
$$

with $\rho_{i}$ the density and $U_{i}$ the mean velocity at the nozzle as $U_{i}=\frac{Q_{i}}{A_{i}}$. The indices $g$ and $l$ refer respectively to gas and liquid. Considering the very low liquid velocity, the instabilities are driven by the gas velocity. $M$ varies here between 3 and 80 .

We consider a Weber number based on the average exit velocities and the liquid inner diameter [21] as We $=\rho_{g}\left(U_{g}-U_{l}\right)^{2} d_{l} / \sigma$ where $\sigma=72 \mathrm{mN} / \mathrm{m}$ is the air-water surface tension. We 
TABLE I. Different magnifications used.

\begin{tabular}{lll}
\hline \hline Magnification & 0.77 & 3.3 \\
Field of view $\left(\mathrm{mm}^{2}\right)$ & $15 \times 23$ & $4 \times 4$ \\
Depth of field $(\mathrm{mm})$ & 6 & 4 \\
Pixel size $(\mu \mathrm{m})$ & 26 & 6 \\
\hline \hline
\end{tabular}

varies between 26.9 and 618 , which means that the initial breakup process is not expected to be governed by surface tension. The liquid flow rate (with $\mathrm{Re}_{l}=1200$ ) being fixed, only the gas flow rate is varied. Thus, only one nondimensional parameter $\left(\mathrm{Re}_{g}\right.$ or $\left.M\right)$ is needed to reflect the range of experimental conditions explored. We choose to discuss results along variations of $\operatorname{Re}_{g}$ and give critical values with $M$ for information and comparison purposes.

This study focuses on purely longitudinal gas co-flow, but the gas nozzle is designed with the ability to add a tunable amount of angular momentum to the gas [Fig. 1(b)], whose consequences will be briefly discussed in Sec. V. The total gas flow rate is then measured as $Q_{g}=Q_{n s}+Q_{s w}$ where $Q_{n s}$ and $Q_{s w}$ are respectively the flow rate of inlets that are perpendicular to the gas nozzle axis and off axis from it. To quantify it, we use the swirl ratio defined as

$$
\mathrm{SR}=\frac{Q_{s w}}{Q_{n s}}
$$

varying from 0 to 1 in this study, for fixed momentum ratios based on the total gas flow rate. The swirl adds an extra control parameter on the spray.

\section{B. High-speed back-lit imaging}

Different experimental challenges arise in observing instabilities along the intact part of the liquid jet. The length of the liquid core, where instabilities might appear, is of the order of several millimeters and they only exist during a time of the order of milliseconds. High spatial and temporal resolution shadowgraphy addresses these challenges and aims at better describing the growth and acceleration of interfacial perturbations in coaxial two-fluid atomization. The resolution used varied between 6 and 26 microns/pixel, depending on the magnification considered, allowing a maximum frame rate of $10 \mathrm{kHz}$, which is well suited to track the interface motion. Due to the fast temporal scales of the process studied, a low exposure time was chosen $(285 \mathrm{~ns})$ to prevent blurred motion.

The spatial characteristics of the process change with momentum ratio $M$. When $M$ increases (by increasing $U_{g}$, as $U_{l}$ remained constant throughout the study), turbulence intensity in the gas phase increases, which creates smaller structures on the interface (see Fig. 2). The atomization efficiency increases, which implies a decrease of the intact length. To adapt to this, we took measurements with two sets of lenses, one yielding a magnification of 0.77 for low values of $M$, and another one with a magnification of 3.3, for the high values of $M$ (typically $M>12$ ). The intermediate range of momentum ratios were imaged with both configurations to confirm the independence of the results to the imaging optics used. The characteristics of each imaging setup are given in Table I.

A background image is used to normalize each shadow graph [21]. This yields almost binary images, with light intensity values close to 1 for the liquid (excluding small liquid inclusions such as droplets, which have intermediate values, but are not the object of study here) and around 0 for the gas, as shown on Fig. 2 (where the scale goes from white to black in the range 0-1; corresponding videos can be found in the Supplemental Material [27]). Simple thresholding then distinguishes the presence of the liquid intact jet, allowing the detection of the interface. Because of the turbulent nature of the process, we collect multiple image sequences for each configuration and parameter set, yielding about 70000 images that were confirmed to ensure complete statistical convergence of the turbulence variables computed. 

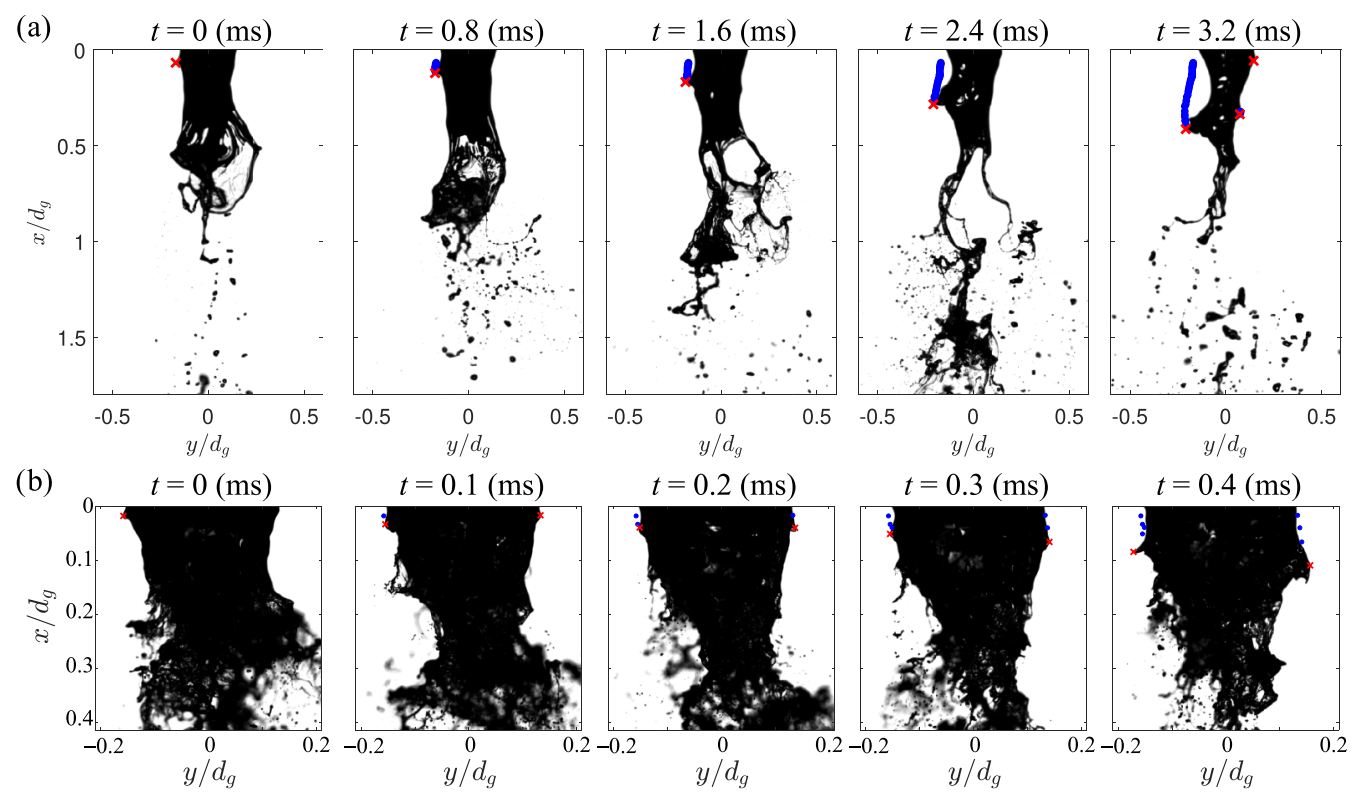

FIG. 2. Two images series displaying the tracking of interfacial perturbations along the liquid jet, with $\operatorname{Re}_{g}=21300(M=5.2)$ and a magnification of 0.77 (a) and $\operatorname{Re}_{g}=69400(M=55)$ and a magnification of 3.3 (b). The red crosses represent the perturbations found on the displayed frame, while the blue dots correspond to the locations detected on previous frames, highlighting the trajectories built by the algorithm. Videos at the corresponding conditions can be found in the Supplemental Material [27].

\section{ANALYSIS}

\section{A. Instability tracking}

To study the different instabilities of the interface, as they develop along the surface of the liquid jet, we chose a Lagrangian approach, tracking their position with time. We use a tracking method similar to [26] and [13]. This tracking analysis is applied independently on both cross sections of the interface (as the image focal plane intersects the liquid jet it defines two contours). First, the location of the edge of the jet is found using the binary image. Then, the interfacial perturbations are located by finding the local maxima of the line from edge detection. A nearest neighbor tracking code is applied to the image sequences, due to the sparse nature of this tracking problem, yielding the coordinates of the interfacial perturbations over time. Finite difference is applied on the trajectories to obtain the velocities of the interfacial perturbations.

Two examples of the Lagrangian trajectories obtained are represented in Fig. 2 for $\operatorname{Re}_{g}=21300$ $(M=5.2)$ (a) and $\operatorname{Re}_{g}=69400(M=55)(\mathrm{b})$. As the characteristic time scales widely differ between these conditions, some frames in the sequence are skipped in (a), for better visibility. One can observe that for high values of $\operatorname{Re}_{g}$ [Fig. 2(b)] the spatiotemporal scales of the process are much smaller than for low $M$ values [Fig. 2(a)]. This illustrates the need for a multiscale analysis, as is conducted in this study.

\section{B. Eulerian velocity}

The interfacial perturbations travel along the intact length of the jet as they are advected by the high speed gas. The average velocity at each location along the longitudinal axis of the liquid jet is computed by Eulerian conditioning of the tracks $\left\langle\mathbf{v}^{E}(x)\right\rangle$. The Lagrangian location along the spray axis is discretized in bins, where $x$ corresponds to the center of a bin. $\left\langle\mathbf{v}^{E}(x)\right\rangle$ is then computed as 

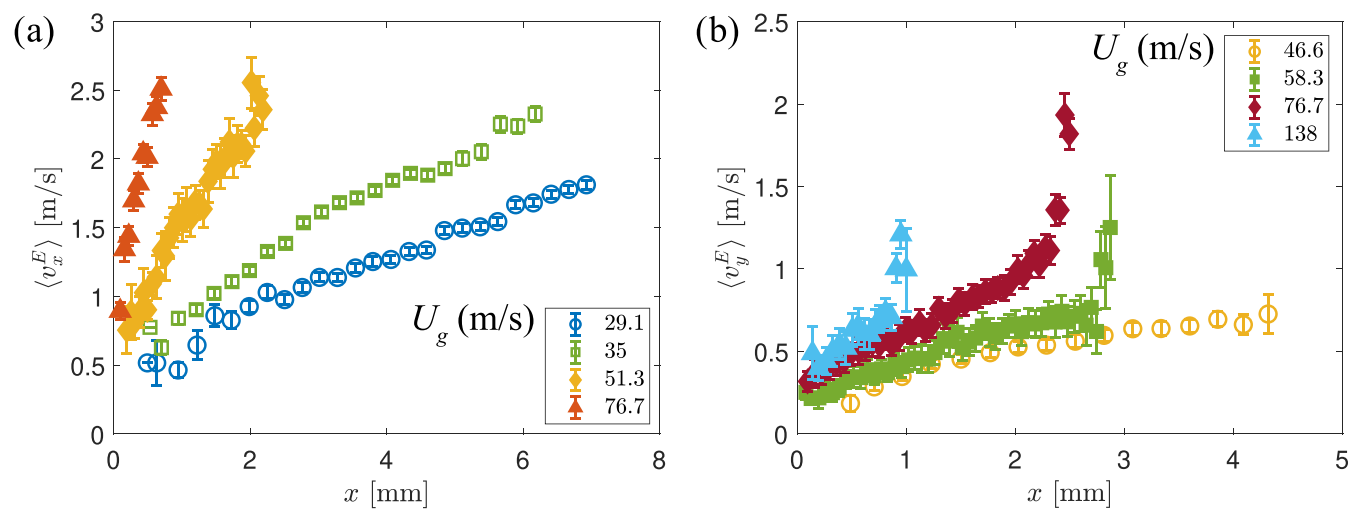

FIG. 3. Evolution of the local time-average longitudinal (a), and transverse (b), velocity of the interfacial perturbations advected along liquid core. The velocity is obtained from an Eulerian conditioning of the Lagrangian trajectories with binning on the longitudinal axis $x$. Each symbol corresponds to an average of the velocities passing through a bin, whereas the error bars represent its convergence error (standard deviation divided by the square root of the number of sample points). The empty symbols are obtained using a magnification of 0.77 , the filled symbols using a magnification of 3.3 ; only a few curves are presented for visibility.

the average of the velocities for interfacial perturbations measured at the locations contained in the bin centered on $x$.

The longitudinal and transverse Eulerian velocities $\left\langle v_{x}^{E}\right\rangle$ and $\left\langle v_{y}^{E}\right\rangle$ are represented in Fig. 3. Both velocities increase along $x$, which means that the interface accelerates downstream. Note that, within the range explored, we did not observe constant velocity fluid perturbations, which would correspond to interface motion driven by the gas co-flow (constant velocity in the potential cone). This velocity gradient seems to be about constant. Increasing the gas velocity $U_{g}$ increases the slopes of $\left\langle\mathbf{v}^{E}(x)\right\rangle$. At high values of $x$, a large increase of the transverse velocity $\left\langle v_{y}^{E}\right\rangle$ appears, which is due to sudden deformations of the interface after the perturbations have progressively accelerated. This leads to the formation of elongated ligaments, bags, or a direct breakup event.

\section{GRADIENT OF THE LOCAL AVERAGE VELOCITY}

The evolution of $v_{x}(x)$ and $v_{y}(x)$ are fitted using a linear function from the data of Fig. 3, restricted to the range where it is linearly accelerated (excluding the large deformation or breakup events). The slopes obtained are presented as a function of the gas velocity $U_{g}$ in Fig. 4. Increasing the gas velocity leads to larger spatial gradients of the average local velocity, related to the perturbations being accelerated faster by the faster gas. We can observe that the increase of the transverse velocity $\frac{\partial\left\langle v_{y}^{E}\right\rangle}{\partial x}$ is also linearly proportional to the gas velocity $U_{g}$ over the whole range explored, as can be seen with the linear fit intersecting 0 in Fig. 4(b).

The evolution of the longitudinal spatial velocity gradient seems to present several regimes with $U_{g}$, which can be analyzed in terms of the nondimensional parameters that control the process. The velocity gradient is made dimensionless using $\frac{d_{l}}{U_{g}}$, and plotted versus the gas Reynolds number in Fig. 5(a). Two regimes appear: when $\operatorname{Re}_{g}<45000$ (this threshold is approximately $M=25$, when expressed in terms of momentum ratio), we can observe that $\frac{\partial\left\langle v_{x}^{E}\right\rangle}{\partial x} \frac{d_{l}}{U_{g}}$ evolves linearly with $\operatorname{Re}_{g}$, whereas when $\operatorname{Re}_{g}>45000$ it reaches a plateau. This corresponds to a quadratic then linear evolution along the gas velocity $U_{g}$. As the liquid velocity is kept constant, this threshold corresponds to $U_{g}=76 \mathrm{~m} / \mathrm{s}$. 

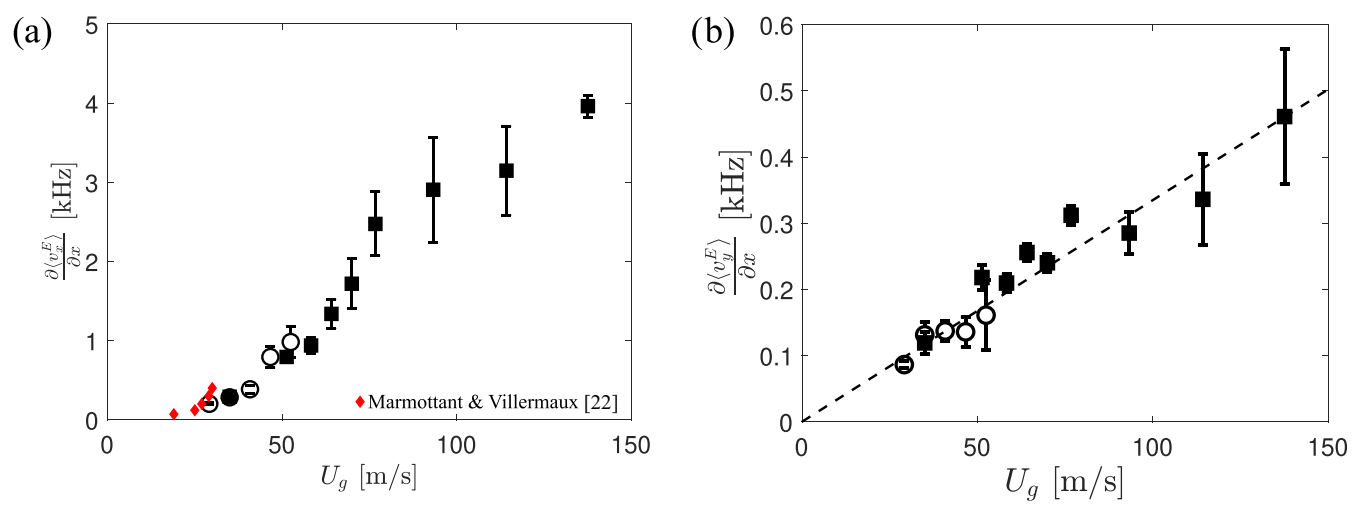

FIG. 4. Evolution of the spatial gradient of the local average longitudinal velocity (a), and transverse velocity (b), with the gas velocity $U_{g}$. The dashed line in (b) is a linear fit with a $0 y$ intercept. The empty symbols are obtained using a magnification of 0.77 , the filled symbols using a magnification of 3.3. The red diamonds on (a) are extracted from data obtained by Marmottant and Villermaux [22].

\section{DISCUSSION}

The first observation from this study is that both the longitudinal and transverse average velocities, conditioned by their axial position (Eulerian), evolve linearly with the downstream distance $x$. Typical interfacial perturbations have been observed to travel at a constant velocity, namely the convective velocity: $U_{c}=\frac{\sqrt{\rho_{l}} U_{l}+\sqrt{\rho_{g}} U_{g}}{\sqrt{\rho_{l}}+\sqrt{\rho_{g}}}[9,14]$. This situation corresponds to waves, created by instabilities, that propagate downstream at a given celerity. However, here, for coaxial atomization at high gas Reynolds numbers, once the perturbations are formed, their crests seem to be immediately exposed to strong hydrodynamical forces and they should be treated as Lagrangian objects: the crests get accelerated, leading to a growing velocity along the liquid core length. This increase of velocity has already been reported by Marmottant and Villermaux [22], using double exposure imaging to compute instantaneous velocity measurements, and by Gutteridge [26], who averaged interfacial velocities in a $3 \mathrm{~mm}$ window using an image correlation method. These results were obtained for a small range of momentum ratios $0.5<M<10$ for [22] and for planar atomization
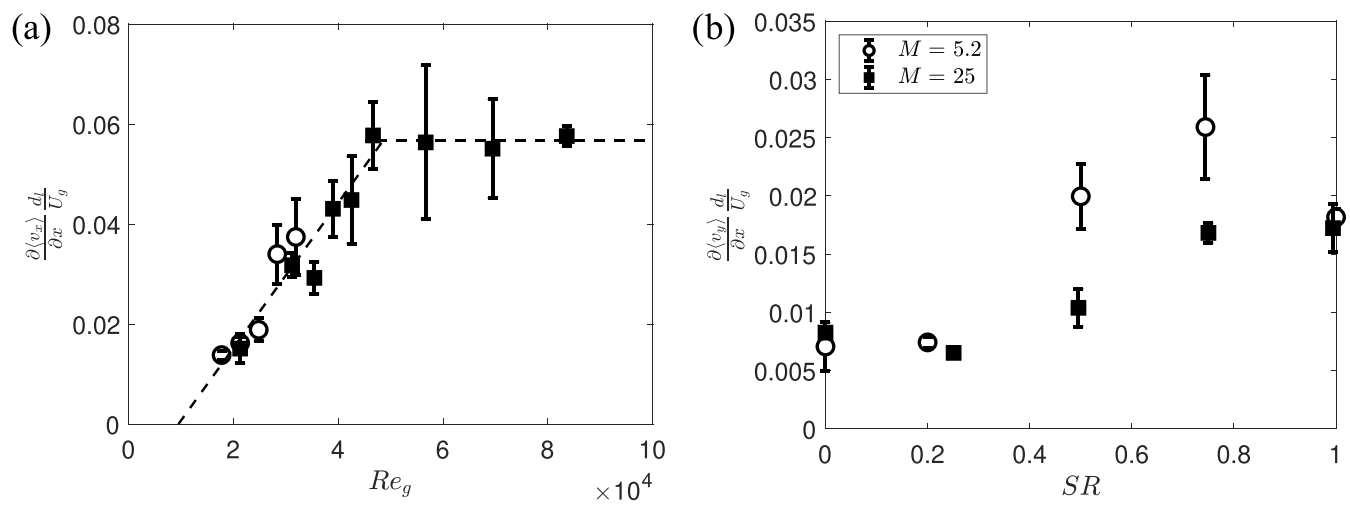

FIG. 5. Nondimensionalization of the spatial velocity gradient, for the longitudinal (a) and transverse component (b). (a) Evolution with Reynolds number for $\mathrm{SR}=0$; (b) evolution $\mathrm{SR}$ at $\operatorname{Re}=21300$ and 46600 , corresponding to $M=5.2$ and 25 . The empty symbols are obtained using a magnification of 0.77 , the filled symbols using a magnification of 3.3 . 
at lower Reynolds numbers for [26], and the present study confirms these findings expanding them to a wider range of parameters.

Thanks to the extended range of momentum ratio, the present study discovered two regimes for the longitudinal velocity gradient, depending on the gas velocity (momentum ratio or Reynolds number). In the lower range of gas Reynolds number $\frac{\partial\left\langle v_{x}^{E}\right\rangle}{\partial x} \frac{d_{l}}{U_{g}} \propto \operatorname{Re}_{g}$ [see Fig. 5(a)], whereas for higher gas Reynolds number another regime appears where $\frac{\partial\left\langle v_{x}^{E}\right\rangle}{\partial x} \frac{d_{l}}{U_{g}}$ reaches a plateau, which means that the gradient of velocity is proportional to the gas velocity [see Fig. 4(a)]. The transition is observed at $\operatorname{Re}_{g}=45000$, corresponding to $M \approx 25$.

Marmottant and Villermaux [22] also observed a regime where the spatial velocity gradient of the longitudinal velocity grows with $\mathrm{Re}_{g}$. Despite a very similar coaxial jets configuration, they found that $\frac{\partial\left\langle v_{x}^{E}\right\rangle}{\partial x} \propto U_{g}^{3}$ so that $\frac{\partial\left\langle v_{x}^{E}\right\rangle}{\partial x} \frac{d_{l}}{U_{g}} \propto \mathrm{Re}_{g}^{2}$ [22], in the range explored. Their experimental results are displayed in Fig. 3 with red symbols, for comparison, and despite the different scaling law, seem to fit reasonably well with the new data presented (they both give a similar trend within the same order of magnitude, even in linear plots). While the experimental configurations are very similar, the liquid Reynolds number $\mathrm{Re}_{l}$ in that experiment was about three times higher, and thus above the transitional Reynolds for pipe flow. The turbulence present in the liquid may explain the difference in the scaling law of the interface perturbation velocity, as it could seed the interface with perturbations at different wave numbers, which could then be temporarily excited by the gas co-flow. Additionally, they found that the interface velocity gradient was inversely proportional to $U_{l}$. While beyond the scope of the present article, we compared the spatial gradient values for three different liquid Reynolds numbers, $\operatorname{Re}_{l}=[1200,2400,5900]$ at $\operatorname{Re}_{g}=2.1 \times 10^{4}$, and found

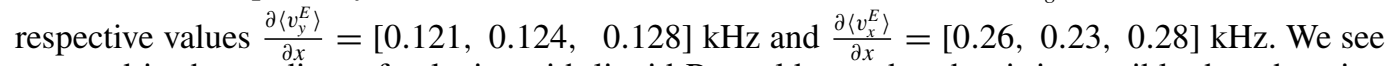
no trend in the gradient of velocity with liquid Reynolds number, but it is possible that changing the liquid injection would change the scaling law for the interface perturbation velocity gradient. The preliminary study made here on the effect of $\operatorname{Re}_{l}$ did not lead to a change of the velocity gradient, however future investigation at a wide range of liquid Reynolds numbers, with accurate description of the laminar or turbulence state at injection, in a wide range of gas Reynolds number would be necessary to conclude on this topic. A dependence of the velocity gradients on both the gas and liquid Reynolds numbers would then be possible. Note that if the effect of $\operatorname{Re}_{l}$ is inversely proportional to the effect of $\mathrm{Re}_{g}$, the use of $M$ as a single parameter would become more relevant, but this remains an open question. In the second regime, a constant spatial gradient of about $0.06 d_{l} / U_{g}$ is found as the nondimensional gradient of velocity. This is reminiscent of scaling laws found for fully developed high Reynolds number fluid mechanics (e.g., asymptotic values of turbulent velocity fluctuations normalized by a mean or forcing velocity in turbulent flows at high Reynolds numbers), hinting that they are purely driven by the gas jet turbulence.

We now take a closer look at the transition threshold between both regimes. When $\operatorname{Re}_{g}<45000$, the large-scale fluctuations, with motions referred to as flapping in the literature, are the dominant factor in interfacial instability and liquid breakup [16]. The meandering (flapping) liquid jet can be seen in Fig. 2(a), and bag (or membrane) breakup is typically observed. Atomization in this range of liquid Reynolds and Weber numbers has been described as membrane breakup [12]. For $\operatorname{Re}_{g}>45000$, while large-scale fluctuations can still exist, the atomization mechanism is through Raleigh-Taylor-type instabilities that develop as elongated ligaments are exposed to large aerodynamic forces [22], and their interface suffers a strong transverse acceleration. This regime is referred to as fiber-type atomization. The $\left(\mathrm{Re}_{l}, \mathrm{We}\right)$ phase diagram of atomization modes presented in [12] shows the border between membrane and fiber atomization to be approximately at $\mathrm{We}=200$ for $\operatorname{Re}_{l}=1000$. In the experiments described here, this corresponds to $\operatorname{Re}_{g}=5 \times 10^{4}$ which is in good agreement with the transition observed at 45000 from the behavior of the velocity gradient. This indicates that the change of regime in the scaling of the interfacial instabilities is related to the different liquid jet topologies shown in [28], and to the transition between atomization modes in coaxial gas-liquid sprays. The agreement between the change in atomization regimes found in the 
literature [12] and the observed transition in the scaling law of the longitudinal gradient of velocity with gas Reynolds number is the main contribution of this paper.

For the range of Reynolds numbers considered, the results seem to indicate a single scaling law for the spatial gradient of the transverse velocity [Fig. 3(b)]. This regime corresponds to a linear growth of the transverse velocity gradient with gas velocity. Data from synchrotron x-ray radiography in [28] shows a very unstable regime of the liquid jet for high momentum ratios (corresponding to the high Reynolds number range), where gas penetrates inside the liquid jet forming a crown around the liquid nozzle rim and making it unstable. These intermittent but very large scale perturbations of the interface can dominate the measurements of the transverse velocity, resulting in larger values of the standard deviation in the transverse velocity compared to the longitudinal values [see error bars in Fig. 3(b)]. This mechanism does not significantly affect the longitudinal velocity but may inhibit the existence (or cloud the detection) of a second regime for the transverse velocity gradient. Further studies at high Reynolds number using synchrotron imaging are needed to further understand the relationship between the formation of a liquid crown and the regime in transverse velocity of the interface.

While beyond the scope of the present contribution, the effect of gas swirl is briefly explored. Adding angular momentum to the gas co-flow can significantly modify the flow fields for both fluids in coaxial atomization, and therefore alter the development of the interfacial instabilities that are responsible for liquid breakup and the formation of the spray, and for the transport of the droplets once formed. Figure 5(b) shows the evolution of the transverse velocity gradient with swirl ratio for two constant values of the gas momentum ratio $(M=5.2$ and $M=25)$. When the swirl ratio is small, $\mathrm{SR}<0.5$, it does not impact the dynamics of the jets, leading to the definition of the critical swirl number [29]. At higher values of the swirl ratio, addition of angular momentum tends to increase the transverse velocity gradient. At the highest swirl explored, $\mathrm{SR}=1$, the interface velocity gradient is smaller than for $\mathrm{SR}=0.75$, especially at the lower momentum ratio. Similar behavior was found by [21] for several metrics of the spray near-field. This is hypothesized to be caused by vortex breakdown inside the nozzle, at high swirl and low gas Reynolds number [21]. The gas velocity inside the nozzle, and in particular the rate of strain, is not sufficient to stretch the vorticity and contain the angular momentum of the gas into a single stable vortex inside the nozzle. We present only data for the transverse velocity gradient, but similar observations are made for the longitudinal component.

\section{CONCLUSION}

This study focuses on the interfacial instabilities that lead to the initial breakup of a liquid jet by a high speed gas coaxial flow. Using a tracking algorithm to determine the velocity of the interfacial perturbations, followed by Eulerian conditioning, we show that the local average velocity of the interface accelerates downstream along the jet. The growth rate, namely the spatial velocity gradient, is determined by the gas velocity, for the high momentum ratio range considered, where the liquid velocity is insignificant. The transverse gradient of velocity evolves linearly with gas velocity, while the longitudinal component shows two regimes: for $\operatorname{Re}_{g}<45000$, a quadratic scaling law is observed, followed by a linear scaling at higher gas Reynolds numbers. These two regimes of the advection velocity for the interfacial perturbations are related with differences in breakup mode, namely with membrane and fiber-type atomization. The latter regime leads to a nondimensional spatial gradient independent of the gas Reynolds number, reminiscent of fully developed turbulence scaling. The characteristics of interfacial perturbation have been shown to be a determining factor for spray metrics in atomization, and their change of regime is likely to be responsible for the different atomization modes described in the literature. The existence of two (respectively one) regimes for the longitudinal (respectively transverse) velocity gradients behavior with respect to the gas Reynolds number are highlighted here. Complementary studies at high gas Reynolds numbers, in the unstable crown regime of the liquid core, using synchrotron x-ray radiography, as well as 
transitional and turbulent regimes of the liquid jet, would enable more a complete understanding and modeling of the interfacial advection velocity and, ultimately, atomization mechanisms.

\section{ACKNOWLEDGMENTS}

This work was sponsored by the Office of Naval Research (ONR) as part of the Multidisciplinary University Research Initiatives (MURI) Program, under Grant No. N00014-16-1-2617. The views and conclusions contained herein are those of the authors only and should not be interpreted as representing those of ONR, the U.S. Navy, or the U.S. Government.

[1] A. H. Lefebvre, Atomization and Sprays (Hemisphere, New York, 1989).

[2] G. P. Sutton and O. Biblarz, Rocket Propulsion Elements (Wiley, New York, 2016).

[3] B. Boust, Q. Michalski, A. Claverie, C. Indiana, and M. Bellenoue, Characterization of liquid impinging jet injector sprays for bi-propellant space propulsion: comparison of pdi and high-magnification shadowgraphy, in Ilass Europe: 28th European Conference on Liquid Atomization and Spray Systems (Universitat Politècnica de València, Valencia, Spain, 2017), pp. 488-495.

[4] B. Sandstede and A. Scheel, Absolute and convective instabilities of waves on unbounded and large bounded domains, Phys. D (Amsterdam, Neth.) 145, 233 (2000).

[5] D. Fuster, G. Agbaglah, C. Josserand, S. Popinet, and S. Zaleski, Numerical simulation of droplets, bubbles and waves: state of the art, Fluid Dyn. Res. 41, 065001 (2009).

[6] P. G. Drazin, Kelvin-helmholtz instability of finite amplitude, J. Fluid Mech. 42, 321 (1970).

[7] J.-P. Matas, S. Marty, M. S. Dem, and A. Cartellier, Influence of Gas Turbulence on the Instability of an Air-Water Mixing Layer, Phys. Rev. Lett. 115, 074501 (2015).

[8] M. R. Ansari and H. Nariai, Experimental investigation on wave initiation and slugging of air-water stratified flow in horizontal duct, J. Nucl. Sci. Technol. 26, 681 (1989).

[9] D. Fuster, J.-P. Matas, S. Marty, S. Popinet, J. Hoepffner, A. Cartellier, and S. Zaleski, Instability regimes in the primary breakup region of planar coflowing sheets, J. Fluid Mech. 736, 150 (2013).

[10] A. Aliseda, E. J. Hopfinger, J. C. Lasheras, D. M. Kremer, A. Berchielli, and E. K. Connolly, Atomization of viscous and non-newtonian liquids by a coaxial, high-speed gas jet. experiments and droplet size modeling, Int. J. Multiphase Flow 34, 161 (2008).

[11] C. M. Varga, J. C. Lasheras, and E. J. Hopfinger, Initial breakup of a small-diameter liquid jet by a high-speed gas stream, J. Fluid Mech. 497, 405 (2003).

[12] J. C. Lasheras and E. J. Hopfinger, Liquid jet instability and atomization in a coaxial gas stream, Annu. Rev. Fluid Mech. 32, 275 (2000).

[13] G. Singh, A. Kourmatzis, A. Gutteridge, and A. R. Masri, Instability growth and fragment formation in air assisted atomization, J. Fluid Mech. 892, A29 (2020).

[14] S. Marty, J.-P. Matas, and A. Cartellier, Study of a liquid-gas mixing layer: Shear instability and size of produced drops, C. R. Mec. 341, 26 (2013).

[15] M. K. Awasthi and G. S. Agrawal, Viscous potential flow analysis of Kelvin-Helmholtz instability of cylindrical interface, Int. J. Appl. Math. Comput. 3(2), 131 (2011).

[16] J.-P. Matas, A. Delon, and A. Cartellier, Shear instability of an axisymmetric air-water coaxial jet, J. Fluid Mech. 843, 575 (2018).

[17] S. Kooij, R. Sijs, M. M. Denn, E. Villermaux, and D. Bonn, What Determines the Drop Size in Sprays? Phys. Rev. X 8, 031019 (2018).

[18] C. Dumouchel, On the experimental investigation on primary atomization of liquid streams, Exp. Fluids 45, 371 (2008).

[19] M. Gorokhovski and M. Herrmann, Modeling primary atomization, Annu. Rev. Fluid Mech. 40, 343 (2008).

[20] J. C. Lasheras, E. Villermaux, and E. J. Hopfinger, Break-up and atomization of a round water jet by a high-speed annular air jet, J. Fluid Mech. 357, 351 (1998). 
[21] N. Machicoane, G. Ricard, R. Osuna-Orozco, P. D. Huck, and A. Aliseda, Influence of steady and oscillating swirl on the near-field spray characteristics in a two-fluid coaxial atomizer, Int. J. Multiphase Flow 129, 103318 (2020).

[22] P. Marmottant and E. Villermaux, On spray formation, J. Fluid Mech. 498, 73 (2004).

[23] A. Delon, A. Cartellier, and J.-P. Matas, Flapping instability of a liquid jet, Phys. Rev. Fluids 3, 043901 (2018).

[24] Y. Ling, D. Fuster, G. Tryggvason, and S. Zaleski, Spray formation: An inverse cascade, arXiv: $1511.04234 \mathrm{v} 1$.

[25] F. Baillot, J.-B. Blaisot, G. Boisdron, and C. Dumouchel, Behaviour of an air-assisted jet submitted to a transverse high-frequency acoustic field, J. Fluid Mech. 640, 305 (2009).

[26] N. Machicoane, J. K. Bothell, D. Li, T. B. Morgan, T. J. Heindel, A. L. Kastengren, and A. Aliseda, Synchrotron radiography characterization of the liquid core dynamics in a canonical two-fluid coaxial atomizer, Int. J. Multiphase Flow 115, 1 (2019).

[27] See Supplemental Material at http://link.aps.org/supplemental/10.1103/PhysRevFluids.6.084302 for videos illustrating the liquid core destabilization at $\operatorname{Re}_{g}=21300(M=5.2)$ and at $\operatorname{Re}_{g}=69400$ $(M=55)$.

[28] A. Gutteridge, An analysis of liquid jet instabilities formed from coaxial air-blast atomization of viscous fuels, Master's thesis, University of Sydney (2019).

[29] E. J. Hopfinger and J. C. Lasheras, Explosive breakup of a liquid jet by a swirling coaxial gas jet, Phys. Fluids 8, 1696 (1996). 